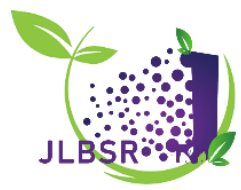

\title{
Serum Asprosin Levels in Women with Polycystic Ovary Syndrome in Duhok City, Kurdistan Region of Iraq
}

\author{
Mohammed Rashid Ameen*1, Dhia Mustafa Sulaiman², Khalida Hassan Muho ${ }^{3}$ \\ ${ }^{I}$ Nursing Department, Akre technical Institute, Duhok Polytechnic University, Iraq, moheme.rashid@gmail.com \\ ${ }^{2}$ Duhok technical Institute, Duhok Polytechniy University,Iraq, dhiamizori@gmail.com \\ ${ }^{3}$ Obstetric and gynecological department, College of medicine, University of Duhok, Iraq,drkhm75@gmail.com \\ *Correspondance: moheme.rashid@gmail.com
}

\begin{abstract}
Asprosin is a novel peptide hormone produced and secreted by white adipose tissues. Asprosin associated with insulin resistance and promotes hepatic glucose production. Previous studies showed that serum asprosin was raised in the general population with polycystic ovary syndrome (PCOS). However, there were studies supporting the opposite. Also, there were studies that showed the highest levels of asprosin was due to insulin resistance, as well as in type 2 diabetes patients. PCOS is one of the metabolic disorders related to insulin resistance. Therefore, the current study aims to evaluate the levels of asprosin in the blood serum of women with PCOS compared to the healthy women who resident in Duhok in the Kurdistan Region of Iraq. A cross-sectional study was conducted from 20th of June, 2020 to 11th of January, 2021 at Obstetrics and Gynecology Hospital and Mazi medical clinics. Serum asprosin level was determined in 75 women with PCOS (18-44 years) and 96 healthy women. SPSS software was utilized for analyzing the study data. The (means \pm SD) of demographic parameters (body mass index (BMI) and waist circumference (WC)) in women with PCOS were significantly highest in comparison to healthy women. The biochemical parameters (serum asprosin, fasting blood sugar (FBS), fasting insulin (FI), total cholesterol (TC), and triglyceride (TG)) in women with PCOS also were remarkably higher compared to healthy women with the exception of high-density lipoprotein- cholesterol (HDL-C). The current data show that serum asprosin variance significantly between WC, BMI, FBS, FI, TC, TG and HDL-C. The study confirms that serum asprosin in women with PCOS was higher than in the healthy women. In addition in women with PCOS it was found that serum asprosin was positively correlated with BMI, WC, FBS, FI, HOMAIR, TC and TG $(\mathbf{P}<0.05)$. Except, HDL-C was negatively correlated with serum asprosin $(\mathbf{P}<0.01)$.
\end{abstract}

Keywords: Asprosin, PCOS, Insulin resistance, Metabolic disorders, Duhok.

Received: April 13 $3^{\text {th }}, 2021 /$ Accepted: June $16^{\text {th }}, 2021 /$ Online: June $26^{\text {th }}, 2021$.

\section{INTRODUCTION}

Polycystic ovary syndrome (PCOS) is the most prevalent endocrine and metabolic disease in women with an incidence of up to $17.8 \%$, with hyperandrogenism, abnormal menstrual periods and polycystic ovaries (Witchel et al., 2019).

In women with polycystic ovary syndrome, obesity and an aberrant metabolic profile are prevalent and about 50-70\% of them are insulin resistant (Dumesic et al., 2015). While the majority of women with PCOS compensate for their insulin resistance (IR), a significant proportion of them have impaired beta-cell function, resulting in glucose sensitivity, which increases their risk of rising type two diabetes mellitus, regardless of their body mass index (BMI) or age (Alan et al., 2019).

Additionally, women with PCOS are at an increased risk of rising dyslipidemia and hypertension, which are associated with an increased prevalence of metabolic syndrome (Amiri et al., 2020; Özkan et al., 2020).

The PCOS etiology remains unknown; although it is believed to be multifactorial. Hyperinsulinemia and hyperandrogenism in PCOS are strongly linked, but the processes underlying their relationship with polycystic ovary syndrome are not well understood (Rosenfield and Ehrmann, 2016; Escobar-Morreale, 2018).

Asprosin is a novel identified peptide hormone produced and secreted by white adipose tissue. Asprosin enhances the release of glucose from the

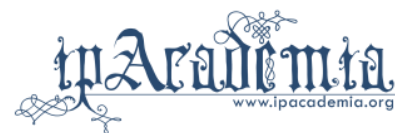


liver by stimulating the guanine nucleotide-binding proteinAdenosine 3',5'-cyclic monophosphate - protein kinase A (G protein-cAMP-PKA) pathway (Romere et al., 2016; Duerrschmidi et al., 2017).

Recently, researchers have conducted several studies on asprosin and they were observed that asprosin levels were rises relative to controls in subjects with type two diabetes. Also, asprosin levels were correlated directly with insulin resistance (Wang et al., 2018; Zhang et al., 2019).

Obesity, PCOS, diabetes, and cardiovascular disease (CVD) are all metabolic diseases that are caused by a breakdown to natural metabolic processes; pose a significant risk to human health. Thus, the discovery of asprosin was a key field of research for the treatment of multiple metabolic diseases associated with insulin resistance. Recent research has established that asprosin has a critical and complex function in metabolism and metabolic diseases (Yuan et al., 2020).

To conduct more studies on asprosin and its effect on metabolic disorders, which include insulin resistance and PCOS, this study was conducted to evaluate serum asprosin levels in women with PCOS in the Kurdistan Region of Iraq. So this study considered as one of the new approaches for studying women with PCOS in our country.

\section{MATERIALS AND METHODS}

\section{A. Subjects and study design}

To achieve the aims of the study, a cross-sectional method was used. This study was performed at Obstetrics and Gynecology Hospital, Mazi Private Laboratory, and Mazi medical clinics in Duhok, Kurdistan Region-Iraq. The study was established from June $20^{\text {th }}, 2020$ to $11^{\text {th }}$ January 2021. A total of 171 women (with ages ranging from (18-44) years) were selected throughout the period of performing this study. The study recruited 75 women from the study population who fulfilled the PCOS criteria, and 96 women as healthy women.

\section{B. Data collection and research tool}

A questionnaire was designed to elicit the necessary information from participants. After informing each participant about the study and obtaining their consent, the standardised questionnaire was administered via direct interview. Personal information (phone number, age, place of employment, and address), pregnancy history, menstrual history, birth control history, height, weight, BMI, waist circumference, prior gynaecological surgery, chronic diseases related to exclusion criteria, oral hypoglycemic drug history, and insulin therapeutic drug history were included in the questionnaire type.

\section{Inclusion criteria}

\section{Polycystic ovary syndrome group}

PCOS patients were diagnosed according to Rotterdam criteria (The Rotterdam ESHRE/ASRM-sponsored PCOS consensus workshop group, 2004), after excluding other causes of hyperandrogenism and menstrual cycle irregularities. In this study a gynecologist uses Rotterdam criteria in the selection PCOS patients, which states the presence 2 of 3 the following criteria; Firstly, oligo and/or anovulation. Secondly, symptoms of hyperandrogenism (biochemical: total testosterone > $70 \mathrm{ng} / 100 \mathrm{ml}$, androstenedione > 245ng/100ml, dhea-s > 248ng/100ml, clinical signs: acne, hirsutism, acanthosis nigrans). Finally, polycystic ovaries on ultrasonography (ovarian volume 10 $\mathrm{cm} 3$ and / or $>12$ follicles in the antral region in one ovary). In addition, ultrasonography was done by the radiologist to exclude the other causes of hyperandrogenism and menstrual abnormality.

\section{Healthy women group}

The participants in this category were selected among the women who have regular menstrual cycles (25-35 days). Also, there were no endocrine anomalies, no physiological or biochemical signs of androgen excess and normal ultrasonic ovarian morphology.

\section{Exclusion criteria}

The exclusion criteria were attended to subject with the following conditions; Hypothyroidism, renal / liver disease, tumor of androgen-secreting, cushing's syndrome, congenital adrenal hyperplasia, hyperprolactinemia, congestive heart diseases, insulin resistance circumstances like acromegaly, use of medications for decreasing hypertension, dyslipidemia, hyperglycemia, insulin resistance, or obesity also were excluded, as well as the nonfasting patient was excluded in the present study.

\section{E. Anthropometric assessment}

Anthropometric measurements were assessed that include; age, height $(\mathrm{cm})$, weight $(\mathrm{kg})$, and waist circumference (centimeter). Additionally, all subjects' blood pressures were measured after 15-minutes of rest time in the seated position. The BMI value was calculated using the following formula: $\mathrm{BMI}=$ weight $(\mathrm{kg}) /$ square metre of height $\left(\mathrm{m}^{2}\right)$ (Deore et al., 2012).

\section{F. Blood samples collection}

Participants were given directives to attend the Mazi privet laboratory in the morning after overnight fasting for 10 hours. Around 9:30 and 11:30 a.m., samples of blood were obtained. Approximately $10 \mathrm{ml}$ of blood was withdrawn and collected by venipuncture in the BD vacutainer device (in two serum separator tubes). The first tube was allowed for 15 minutes after blood collection, then serum was separated for 8 minutes using a centrifuge at $1000 \mathrm{xg}$, the obtained serum samples were prepared promptly so as to measure lipid profile, FBS, and fasting insulin following standard laboratory procedures using clinical chemistry analyzer Cobas 6000 Roche. The blood from the second tube was held at room temperature for two hours. Then serum was separated for 20 minutes using a centrifuge at $1000 \mathrm{x} \mathrm{g}$. The obtained serum samples from the second tube were stored in deep freeze $(-25)$ for later analysis of serum asprosin using the ELISA technique 


\section{G. Biochemical assessment}

Serum samples were biochemically analyzed using the Autoanalyzer biochemical machine named COBASS series 6000 and ELISA technique in order to obtain sensitive and precise findings. A glucose HK kit was used to determine the level of serum (Reference number: 04404483190 Roche/Germany). According to the manufacturer protocol instructions, in this case, cholesterol gen 2 (Reference number: 03039773 190, Roche, Germany) was used to detect the serum total cholesterol. Also, A triglyceride kit (Reference number: 20767107 322, Roche, Germany) was used to identify the serum triglyceride. Moreover, An HDLCholesterol gen.4 kit (Roche) was used to measure serum high-density lipoprotein-cholesterol. Reference number: 07528566 190. Furthermore, the insulin kit is used to monitor and evaluate fasting insulin levels (Reference number: 12017547 122) also, each test participant was assessed for insulin resistance using the (HOMA-IR) = fasting insulin $(\mu \mathrm{U} / \mathrm{ml}) *$ fasting glucose $(\mathrm{mg} / \mathrm{dl}) / 405$ (Matthews et al., 1985).

\section{H. Serum asprosin assessment}

Human Asprosin ELISA kit with the catalog No.:MBS7606420, sensitivity: 0.938ng/ml (from MyBioSource, Inc. San Diego, USA) was used for quantitative detection of Asprosin in serum in accordance with the instructions of manufactures.

\section{Statistical analysis}

The data were analysed and represented as (mean \pm standard division) using the SPSS programme version 25 . The T-test was performed to compare proportions. In addition, to compare various groups, one-way variance analysis (ANOVA) was utilised. When comparing categorical variables between groups, the Chi-square test was used. The Pearson correlation coefficient was employed to estimate the relationship between variables. ROC curve analysis was used to determine the serum asprosin cut-off point for PCOS prediction. In all experiments, a $\mathrm{p}<0.05$ was statistically significant.

\section{J. Ethical considerations}

The Scientific Committee of Duhok Polytechnic University / Shekhan Technical College and the Ethical Committee of the General Directorate of Health in Duhok have reviewed and approved the study protocols (Code of Ethics:22062020-2).

\section{RESULTS}

\section{A. Demographic and biochemical characteristic features} of study population according to PCOS and healthy women

Demographic characteristics and Biochemical parameters of the population of the study are elucidated in Table 1. Women with PCOS presented significantly higher mean values of BMI, waist circumference (WC), serum asprosin, total cholesterol, serum levels of TG, FBS, fasting insulin, and HOMA-IR in comparison with healthy women $(\mathrm{P}<$ 0.05). Except for HDL-C which was lower in women with PCOS in comparison to healthy women $(\mathrm{P}<0.05)$. On the contrary, there was no significant variation in DBP, SBP and age across the groups $(\mathrm{P}>0.05)$.

Table 1 . The laboratory and demographic characteristics comparison in the study population

\begin{tabular}{|l|l|l|l|}
\hline \multirow{2}{*}{ Variables } & \multicolumn{2}{|c|}{ Mean \pm SD } & \multirow{2}{*}{ P-value } \\
\cline { 2 - 3 } & \multicolumn{1}{|c|}{$\begin{array}{c}\text { PCOS } \\
(\mathrm{n}=75)\end{array}$} & \multicolumn{1}{c|}{$\begin{array}{c}\text { healthy } \\
\text { women(n=96) }\end{array}$} & \\
\hline Age (years) & $27.05 \pm 7.50$ & $25.88 \pm 7.87$ & $\mathrm{NS}$ \\
BMI $(\mathrm{kg} / \mathrm{m} 2)$ & $29.94 \pm 6.82$ & $24.84 \pm 5.62$ & $<0.001^{*}$ \\
WC(cm) & $84.55 \pm 18.01$ & $78.97 \pm 11.49$ & $<0.05^{*}$ \\
SBP. $(\mathrm{mmHg})$ & $113.35 \pm 14.82$ & $114.81 \pm 9.71$ & $\mathrm{NS}$ \\
DBP. $(\mathrm{mmHg})$ & $78.43 \pm 11.21$ & $76.40 \pm 5.58$ & $\mathrm{NS}$ \\
TC $(\mathrm{mg} / \mathrm{dl})$ & $154.73 \pm 34.40$ & $139.72 \pm 25.21$ & $<0.05^{*}$ \\
TG $(\mathrm{mg} / \mathrm{dl})$ & $122.47 \pm 65.39$ & $84.57 \pm 29.78$ & $<0.01^{*}$ \\
HDL- C $(\mathrm{mg} / \mathrm{dl})$ & $41.91 \pm 9.96$ & $56.25 \pm 8.01$ & $<0.05^{*}$ \\
FBS $(\mathrm{mg} / \mathrm{dl})$ & $104.61 \pm 22.52$ & $97.91 \pm 12.64$ & $<0.05^{*}$ \\
Insulin $(\mu \mathrm{H} / \mathrm{mL})$ & $19.26 \pm 9.33$ & $11.26 \pm 4.75$ & $<0.001^{*}$ \\
HOMA-IR & $5.12 \pm 3.28$ & $2.75 \pm 1.34$ & $<0.001^{*}$ \\
Serum asprosin $(\mathrm{ng} / \mathrm{ml})$ & $14.80 \pm 6.67$ & $5.49 \pm 3.42$ & $<0.001^{*}$ \\
\hline
\end{tabular}

t-test was used. A p value of $<0.05$ was considered significant (*). NS: nonsignificant a $\mathrm{p}$ value was $>0.05$ Key:BMI $=$ Bodys MasssIndex, $\mathrm{WC}=$ WaistsCircumference, SBP $=$ Systolic Blood Pressure, DBP $=$ Diastolic Blood Pressure, $\mathrm{TC}=$ Total cholesterol, $\mathrm{TG}=$ Triglyceride, $\mathrm{HDL}-\mathrm{C}=\mathrm{High}$ Density Lipoprotein -Cholesterol, FBS = Fasting Blood Sugar, and HOMA$\mathrm{IR}=$ Homeostatic Model Assessment for Insulin Resistance.

\section{B. Association of asprosin concentration with clinical parameters}

Accordance to the Pearson correlation coefficient (r), there was a remarkable positive relation $(\mathrm{P}<0.001)$ between serum asprosin and age, BMI, Waist circumference, TC, TG, FBS, insulin, and HOMA-IR $(\mathrm{P}<0.05)$ in both groups. Also, there was a significant direct correlation $(\mathrm{P}<0.001)$ between serum asprosin and age, SBP only in the healthy women group $(\mathrm{P}<0.001)$. On contrary, serum asprosin was negatively correlated with HDL-C in both the groups ( $\mathrm{P}$ $<0.05$ ) (Table 2).

Table 2. The correlations between serum asprosin level and possible metabolic risk factors in the study population.

\begin{tabular}{|c|c|c|c|c|}
\hline \multirow[t]{2}{*}{ Variables } & \multicolumn{2}{|c|}{$\operatorname{PCOS}(n=75)$} & \multicolumn{2}{|c|}{$\begin{array}{c}\text { healthy } \\
\text { women }(n=96)\end{array}$} \\
\hline & $r$ & $p$-value & $r$ & p-value \\
\hline Age (years) & .164 & 0.161 & $.590 * *$ & $<0.001$ \\
\hline BMI $(\mathrm{kg} / \mathrm{m} 2)$ & $.322 * *$ & 0.005 & $.671 * *$ & $<0.001$ \\
\hline $\mathrm{WC}(\mathrm{cm})$ & $.369 * *$ & 0.001 & $.611 * *$ & $<0.001$ \\
\hline $\mathrm{SBP}(\mathrm{mmHg})$ & .149 & 0.203 & $.243 *$ & 0.017 \\
\hline $\mathrm{DBP}(\mathrm{mmHg})$ & .079 & 0.499 & .166 & 0.105 \\
\hline $\mathrm{TC}(\mathrm{mg} / \mathrm{dl})$ & $.319 * *$ & 0.005 & $.402 * *$ & $<0.001$ \\
\hline TG (mg/dl) & $.310 * *$ & 0.007 & $.596 * *$ & $<0.001$ \\
\hline HDL- C (mg/dl) & $-.250-*$ & 0.031 & $-.376-* *$ & $<0.001$ \\
\hline FBS (mg/dl) & $.311 * *$ & 0.007 & $.699 * *$ & $<0.001$ \\
\hline Insulin $(\mu \mathrm{U} / \mathrm{mL})$ & $.362 * *$ & 0.001 & $.249 *$ & 0.014 \\
\hline HOMA-IR & $.405 * *$ & $<0.001$ & $.427 * *$ & $<0.001$ \\
\hline
\end{tabular}


Correlations between variables were analyzed by Pearson analysis. (**) Correlation is remarkable at the 0.01 level (2-tailed). (*) Correlation is remarkable at the 0.05 level (2-tailed). Key:BMI $=$ Bodys MasssIndex, WC $=$ WaistsCircumference, $\mathrm{SBP}=$ Systolic Blood Pressure, $\mathrm{DBP}=$ Diastolic Blood Pressure, $\mathrm{TC}=$ Total cholesterol, $\mathrm{TG}=$ Triglyceride, $\mathrm{HDL}-\mathrm{C}=$ High Density Lipoprotein -Cholesterol, FBS = Fasting Blood Sugar, and HOMA$\mathrm{IR}=$ Homeostatic Model Assessment for Insulin Resistance.

\section{The concentration of serum asprosin and PCOS}

Based on concentrations of serum asprosin, all subjects were divided into tertiles (T1: < $4 \mathrm{ng} / \mathrm{mL}$, T2: $4-13.5 \mathrm{ng} / \mathrm{mL}$ and T3: > $13.5 \mathrm{ng} / \mathrm{mL})$. The Demographic characteristics and Biochemical parameters of each category are listed in Table 3. In correspondence to tertiles the parameters like age, waist circumference, BMI, FBG, fasting insulin, $\mathrm{T}$. Cholesterol, TG, and HOMA-IR were increased. Moreover, parameter such as HDL-C decreased in correspondence to tertiles. The number of PCOS patients increased as asprosin concentrations in the tertiles increased, according to the trending of the Chi-square test (Figure 1). Besides that, the major patients with PCOS placed in the third tertile. The receiver operating characteristic (ROC) analysis showed that the cut-off value for serum asprosin to predict PCOS was $(7.476 \mathrm{ng} / \mathrm{ml})(\mathrm{p}>0.001)$. It had an area under the curve (AUC) (0.909), with sensitivity (0.906), and specificity (0.750) (Figure 2).

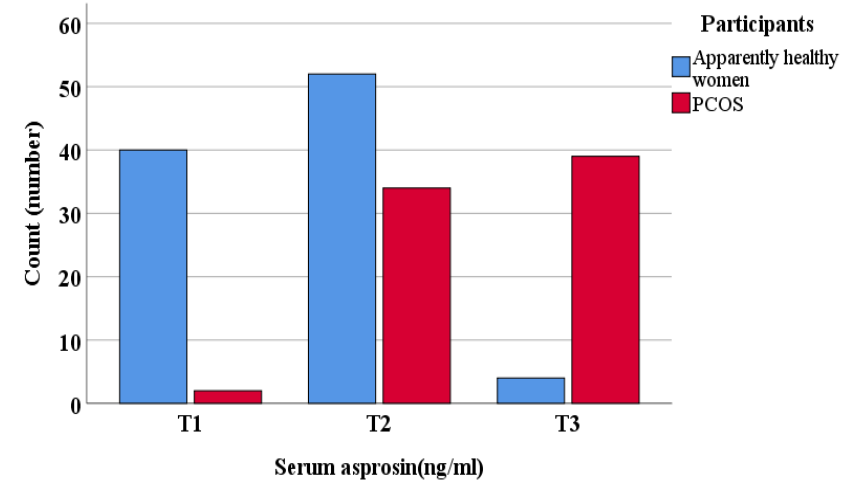

Figure 1.Number of individuals present in each tertile. Most of healthy people were situated in T1 and T2s. However, the number of PCOS women was higher in $\mathrm{T} 2$ and $\mathrm{T} 3$ in comparison to $\mathrm{T} 1$. The most interesting result is that the major number of participants who had PCOS on the T3.

Table 3. Distribution of clinical parameters of participants in different tertiles based on serum concentrations of asprosin in all subjects

\begin{tabular}{|c|c|c|c|c|}
\hline Variables & $\begin{array}{c}(\mathrm{T} 1) \\
\mathrm{N}=42\end{array}$ & $\begin{array}{c}(\mathrm{T} 2) \\
\mathrm{N}=86\end{array}$ & $\begin{array}{c}\text { (T3) } \\
\mathrm{N}=43\end{array}$ & p-value \\
\hline Serum asprosin (ng/ml) & $\begin{array}{l}2.385 \\
0.769\end{array}$ & $\begin{array}{l}8.275 \quad \pm \\
2.666\end{array}$ & $\begin{array}{l}19.194 \quad \pm \\
5.189\end{array}$ & $<0.001$ \\
\hline Age (years) & $\begin{array}{l}21.905 \quad \pm \\
4.023\end{array}$ & $\begin{array}{l}27.233 \quad \pm \\
8.141\end{array}$ & $\begin{array}{l}29.093 \quad \pm \\
7.855\end{array}$ & $<0.001$ \\
\hline BMI (kg/m2) & $\begin{array}{l}22.264 \quad \pm \\
3.522\end{array}$ & $\begin{array}{l}26.602 \quad \pm \\
5.599\end{array}$ & $\begin{array}{l}32.714 \quad \pm \\
6.912\end{array}$ & $<0.001$ \\
\hline $\mathrm{WC}(\mathrm{cm})$ & $\begin{array}{ll}74.679 \quad \pm \\
8.677 & \\
\end{array}$ & $\begin{array}{ll}77.994 & \pm \\
12.365 & \\
\end{array}$ & $\begin{array}{ll}94.837 & \pm \\
16.274 & \\
\end{array}$ & $<0.001$ \\
\hline $\mathrm{SBP}(\mathrm{mmHg})$ & $\begin{array}{l}111.952 \pm \\
10.007\end{array}$ & $\begin{array}{l}114.419 \pm \\
11.708\end{array}$ & $\begin{array}{l}115.837 \pm \\
14.808 \\
\end{array}$ & 0.330 \\
\hline $\mathrm{DBP}(\mathrm{mmHg})$ & $75.643 \pm$ & 76.500 & 80.465 & 0.015 \\
\hline
\end{tabular}

\begin{tabular}{|c|c|c|c|c|}
\hline & 6.362 & 7.295 & 11.583 & \\
\hline $\mathrm{TC}(\mathrm{mg} / \mathrm{dl})$ & $\begin{array}{l}131.452 \pm \\
22.541\end{array}$ & $\begin{array}{l}142.581 \quad \pm \\
25.344\end{array}$ & $\begin{array}{l}168.256 \pm \\
34.767\end{array}$ & $<0.001$ \\
\hline TG (mg/dl) & $\begin{array}{ll}67.952 & \pm \\
21.123 & \\
\end{array}$ & \begin{tabular}{|l|}
$94.791 \pm$ \\
37.106 \\
\end{tabular} & $\begin{array}{l}146.465 \pm \\
66.854\end{array}$ & $<0.001$ \\
\hline HDL- C (mg/dl) & $\begin{array}{ll}57.976 \quad \pm \\
6.848\end{array}$ & $\begin{array}{ll}51.593 \\
10.753\end{array}$ & $\begin{array}{l}38.860 \quad \pm \\
7.220\end{array}$ & $<0.001$ \\
\hline FBS (mg/dl) & $\begin{array}{l}91.643 \pm \\
5.136 \\
\end{array}$ & \begin{tabular}{|l|}
100.000 \\
\pm 13.358 \\
\end{tabular} & $\begin{array}{l}111.535 \pm \\
26.647\end{array}$ & $<0.001$ \\
\hline Insulin $(\mu \mathrm{U} / \mathrm{mL})$ & $\begin{array}{l}9.844 \pm \\
3.002 \\
\end{array}$ & \begin{tabular}{|ll}
14.194 & \pm \\
7.256 & \\
\end{tabular} & $\begin{array}{ll}20.497 \quad \pm \\
9.818\end{array}$ & $<0.001$ \\
\hline HOMA-IR & $\begin{array}{ll}2.231 & \pm \\
0.703 & \end{array}$ & \begin{tabular}{|ll}
3.546 & \pm \\
1.954 &
\end{tabular} & $\begin{array}{l}5.794 \\
3.706\end{array}$ & $<0.001$ \\
\hline
\end{tabular}

One way ANOVA test was used. Data are presented as mean \pm S.D. Key: $\mathrm{BMI}=$ Bodys MasssIndex, $\mathrm{WC}=$ WaistsCircumference, $\mathrm{SBP}=$ Systolic Blood Pressure, DBP $=$ Diastolic Blood Pressure, $\mathrm{TC}=$ Total cholesterol, $\mathrm{TG}=$ Triglyceride, $\mathrm{HDL}-\mathrm{C}=$ High Density Lipoprotein - Cholesterol, FBS = Fasting Blood Sugar, and HOMA-IR= Homeostatic Model Assessment for Insulin Resistance.

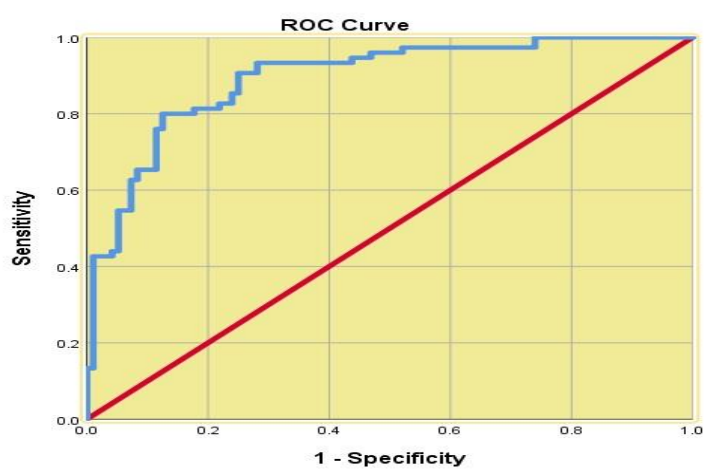

Figure 2. PCOS prediction by utilizing ROC curve analyses on serum asprosin.

\section{Discussions}

Adipose tissue secretes a large number of adipokines that regulate glucose, lipid metabolism and insulin resistance (Coelho et al., 2013). Numerous adipokines such as leptin, ghrelin, asprosin, and irisin have been documented to have modified and influenced insulin secretion and insulin effect in women with PCOS (Polak et al., 2017). Asprosin is a newly known adipokine (Romere et al., 2016). Four independent study groups investigated whether asprosin plays a role in women with PCOS, but their findings were conflicting (Li et al., 2018; Alan et al., 2019; Chang et al., 2019; Deniz et al., 2020). Although Li et al., Alan et al., and Deniz et al. reported that women with PCOS had higher circulating asprosin levels than controls (Li et al., 2018; Alan et al., 2019; Deniz et al., 2020), Chang et al. reported that it was not correlated with the aetiology of PCOS (Chang et al., 2019). Despite this interest, no one has researched the evaluation levels of asprosin in patients with PCOS in the Kurdistan region of Iraq to our knowledge. Therefore, this study was designed to clarify conflicting asprosin results in PCOS. In the current study, our hypothesis was the evaluation of serum asprosin in women with PCOS and if the asprosin can use as a biomarker for diagnosis of PCOS. In this study, we found out that the 
asprosin levels of women with PCOS were significantly higher than in healthy women $(\mathrm{P}$-value $<0.001)$.

Obesity has been associated with abnormal hypothalamicpituitary-ovarian (HPO) axis function through a variety of mechanisms that lead to the development of PCOS (Legro, 2012). Although many people with PCOS are overweight, obese, or centrally obese the impact of obesity on PCOS outcomes is ambiguous (Lim et al., 2013). In the current study, the serum asprosin was positively correlated with BMI and waist circumference in women PCOS and healthy women. As well as, results proved the presence of a significant variation in the mean of serum asprosin according to the BMI and waist circumference in the women with PCOS. Similarly, results show that the presence of a significant difference in the mean of serum asprosin according to the BMI and waist circumference in healthy women.

All the above results illustrated the risk of elevation of serum asprosin in the study population elevated by increasing the BMI and waist circumference. An increase in BMI and waist circumference indicated there were fatty tissues excessive in the body, especially in the abdominal area. Consequently, Romere et al. (2019), claimed that asprosin secretion elevates since the accumulation of these fats in the abdominal area. The results of our study supported by many researchers, they found a positive correlation between serum asprosin and BMI in women with PCOS (Li et al., 2018; Alan et al., 2019; Ugur and Aydin, 2019; Deniz et al., 2020). In contrast with these findings, a cross-sectional study by Jiang et al. (2019) revealed that in PCOS women, serum asprosin was negatively correlated with body mass index.

PCOS is a metabolic disorder that often manifests itself in insulin resistance. Up to $70 \%$ of women with PCOS have insulin resistance. Insulin resistance is a significant factor for the development of PCOS (Rojas et al., 2014; Alan et al., 2019). The current study found a significant difference in fasting insulin, FBS, and HOMA-IR between women with PCOS and healthy women. There was a significant positive association between serum asprosin and FI, FBS, and HOMA-IR in the current study's PCOS women. Similarly, serum asprosin had a significant positive association with FBS, FI, and HOMA-IR in healthy women. Insulin resistance in patients with PCOS may have been caused by a high level of asprosin, which mediates the release of glucose from the liver into circulation (Romere et al., 2016), resulting in an accumulation of glucose in circulation and an excessive secretion of insulin to get the increased circulating glucose to physiologic levels (Rojas et al., 2014). As a result, in current research, increasing the circulating asprosin levels of patients with PCOS could be in order to neutralise the hyperinsulinemia observed in these patients. The fact that patients with PCOS had elevated HOMA-IR values supported this. Another possible explanation for elevated circulating asprosin levels in PCOS patients is the production of asprosin resistance. In other words, high levels of asprosin in patients with PCOS were either not used by the cells or were not metabolised properly as a result of overproduction, and thus could not be removed. Hormones are metabolised either by the liver or the kidneys or by both. However, there is currently no empirical evidence indicating where asprosin is metabolised. Thus, the available evidence suggests that insulin resistance is a significant contributor to hormonal and biochemical defects in PCOS disease.

Additionally, the elevated glucose levels observed in this study may be a result of aprosin's role in the release of glucose from hepatic tissues into circulation, as asprosin is a molecule that facilitates the transport of glucose from the liver to circulation (Romere et al., 2016). The current study's results corroborated those of Li et al. (2018), Alan et al. (2019), and Deniz et al. (2020), but contradicted Jiang et al. (2019), who demonstrated that asprosin was negatively associated with IR and HOMA-IR in women with PCOS and women with IR.

According to the results of this study, there was a significant difference in TC, TG, and HDL-C levels among women with PCOS and healthy women. Whereas, the results of this study are in agreement with the results of recent studies conducted by Pergialiotis et al. (2018), Tsouma et al. (2014), Ghaffarzad et al. (2016). In which they claimed that women with PCOS have lipid defects. Also, PCOS is associated with a variety of lipid forms, including low levels of HDL-C, elevated TG levels and TC levels.

In the present study, there was a strong positive association between serum asprosin and TC and TG levels in women with PCOS. However, the results demonstrated the existence of a significant negative association between HDL-C and serum asprosin in women in both study groups.

In this study, it was found that there was a significant relationship between dyslipidemia and increased asprosin in patients with PCOS. The effect on metabolic parameters was probably due to the effects of asprosin on appetite because it was reported in previous studies that increased asprosin in T2DM patients affected appetite and led to metabolic changes (Li et al., 2018: Zhang et al., 2019). The current study's results corroborated those of Li et al. (2018), Alan et al. (2019), and Deniz et al. (2020), but contradicted that with Jiang et al. (2019), who found that asprosin had a negative correlation with TG and a positive correlation with HDL-C in women with PCOS.

The crude AUC of the asprosin ROC curve for detecting PCOS was 0.909 in the current investigation, and it was regarded to be of good significance, which could be related to the small sample size and non-normal distribution of the examined population. As a result, it may not be an ideal predictor of PCOS diagnosis. So, a large-scale study is recommended to confirm the serum asprosin cut-off value for PCOS diagnosis.

Our study had several limitations; firstly, a cross-sectional design was performed in this study and it was done in a 
single city, so that can be considered as a limitation. Secondly, our sample size was small and could be considered as insufficient for making a general conclusion. Finally, the present study contained only Iraqi-Kurdish women.

\section{CONCLUSIONS}

This study confirms that serum asprosin in women with PCOS was higher than in healthy women. Also, serum asprosin was significantly positively correlated with BMI, waist circumferences, fasting insulin, fasting blood sugar, HOMA-IR, total cholesterol and triglyceride in healthy women and women with PCOS. On contrary, high-density lipoprotein-cholesterol was negatively correlated with serum asprosin in both groups of the study population.

\section{REFERENCES}

Alan, M., Gurlek, B., Yilmaz, A., Aksit, M., Aslanipour, B., Gulhan, I. Mehmet, C., et al. (2019). Asprosin: a novel peptide hormone related to insulin resistance in women with polycystic ovary syndrome. Gynecological Endocrinology,35(3):220-223.

Amiri, M., Tehrani, F.R., Behboudi-Gandevani, S., Bidhendi-Yarandi, R. and Carmina, E. (2020). Risk of hypertension in women with polycystic ovary syndrome: a systematic review, meta-analysis and meta-regression. Reproductive Biology and Endocrinology, 18(1):23.

Chang, C.L., Huang, S.Y., Hsu, Y.C., Chin, T.H., Soong, Y.K. (2019). The serum level of irisin, but not asprosin, is abnormal in polycystic ovary syndrome patients. Scientific Reports, 9(1):6447.

Coelho, M., Oliveira, T., Fernandes, R. (2013). Biochemistry of adipose tissue: an endocrine organ. Archives of Medical Science, 9(2):191-200.

Deniz, R., Yavuzkir, S., Ugur, K., Ustebay, D.U., Baykus, Y., Ustebay, S. Aydin, S. (2020). Subfatin and asprosin, two new metabolic players of polycystic ovary syndrome. Journal of Obstetrics and Gynaecology,41(2):279-284.

Deore, D.N., Surwase, S.P., Masroor, S., Khan, S.T., Kathore, V. (2012). A Cross Sectional Study on the Relationship Between the Body Mass Index (BMI) and the Audiovisual Reaction Time (ART). Journal of Clinical And Diagnostic Research;6(9):1466-8.

Duerrschmid, C., He, Y., Wang, C., Li, C., Bournat, J.C., Romere, C. Saha, P.K., et al. (2017). Asprosin is a centrally acting orexigenic hormone. Nature Medicine, 23(12):1444-1453.

Dumesic, D.A., Oberfield, S.E., Stener-Victorin, E., Marshall, J.C., Laven, J.S., Legro, R.S. (2015). Scientific Statement on the Diagnostic Criteria, Epidemiology, Pathophysiology, and Molecular Genetics of Polycystic Ovary Syndrome. Endocrine Reviews, 36(5):487-525.

Escobar-Morreale, H.F. (2018). Polycystic ovary syndrome: definition, aetiology, diagnosis and treatment. Nature Reviews Endocrinology, 14(5):270-284.

Ghaffarzad, A., Amani, R., Sadaghiani, M., Darabi, M., Cheraghian, B. (2016). Correlation of Serum Lipoprotein Ratios with Insulin Resistance in Infertile Women with Polycystic Ovarian Syndrome: A Case Control Study. International Journal Of Fertility \& Sterility, 10(1):29-35.

Jiang, Y., Liu, Y., Yu, Z., Yang, P., Xie, L., Shang, X. Zhao, S. (2020). Serum asprosin level in different subtypes of polycystic ovary syndrome: a cross-sectional study, PREPRINT (Version 1) available at Research Square.

Legro, R.S. (2012). Obesity and PCOS: implications for diagnosis and treatment. Seminars in reproductive medicine, 30(6):496-506.

Li, X., Liao, M., Shen, R., Zhang, L., Hu, H., Wu, J. Wang, X., et al. (2018). Plasma Asprosin Levels Are Associated with Glucose Metabolism, Lipid, and Sex Hormone Profiles in Females with Metabolic-Related Diseases. Mediators of Inflammation,2018:7375294.
Lim, S.S., Norman, R.J., Davies, M.J., Moran, L.J. (2013). The effect of obesity on polycystic ovary syndrome: a systematic review and meta-analysis. Obesity Reviews, 14(2):95-109.

Matthews, D.R., Hosker, J.P., Rudenski, A.S., Naylor, B.A., Treacher, D.F., Turner, R.C. (1985). Homeostasis model assessment: insulin resistance and beta-cell function from fasting plasma glucose and insulin concentrations in man. Diabetologia,28(7):412-419.

Özkan, S., Yılmaz, Ö.Ç., Yavuz, B. (2020). Increased masked hypertension prevalence in patients with polycystic ovary syndrome (PCOS). Clinical and Experimental Hypertension, 42(8):681-684.

Pergialiotis, V., Trakakis, E., Chrelias, C., Papantoniou, N., Hatziagelaki, E. (2018). The impact of mild hypercholesterolemia on glycemic and hormonal profiles, menstrual characteristics and the ovarian morphology of women with polycystic ovarian syndrome. Hormone Molecular Biology And Clinical Investigation,34(3). 20180002.

Polak, K., Czyzyk, A., Simoncini, T., Meczekalski, B. (2017). New markers of insulin resistance in polycystic ovary syndrome. Journal of Endocrinological Investigation,40(1):1-8.

Rojas, J., Chávez, M., Olivar, L., Rojas, M., Morillo, J., Mejías, J. Calvo, M., Bermúdez, V. (2014). Polycystic ovary syndrome, insulin resistance, and obesity: navigating the pathophysiologic labyrinth. International Journal of Reproductive Medicine,2014:719050.

Romere, C., Duerrschmid, C., Bournat, J., Constable, P., Jain, M., Xia, F., Saha, P.K., et al. (2016). Asprosin, a Fasting-Induced Glucogenic Protein Hormone. Cell, 165(3):566-579.

Rosenfield, R.L., Ehrmann, D.A. (2016). The Pathogenesis of Polycystic Ovary Syndrome (PCOS): The Hypothesis of PCOS as Functional Ovarian Hyperandrogenism Revisited. Endocrine Reviews, 37(5):467-520.

The Rotterdam ESHRE/ASRM-sponsored PCOS consensus workshop group. (2004). Revised 2003 consensus on diagnostic criteria and long-term health risks related to polycystic ovary syndrome (PCOS). Human Reproduction, 19, 41-47.

Tsouma, I., Kouskouni, E., Demeridou, S., Boutsikou, M., Hassiakos, D., Chasiakou, A. Hassiakou, S., et al. (2014). Lipid lipoprotein profile alterations in Greek infertile women with polycystic ovaries: influence of adipocytokines levels. In vivo, 28(5):935939.

Ugur, K., Aydin, S. (2019). Saliva and Blood Asprosin Hormone Concentration Associated with Obesity. International journal of endocrinology, 2019:2521096.

Wang, Y., Qu, H., Xiong, X., Qiu, Y., Liao, Y., Chen, Y., Zheng, Y., et al. (2018). Plasma Asprosin Concentrations Are Increased in Individuals with Glucose Dysregulation and Correlated with Insulin Resistance and First-Phase Insulin Secretion. Mediators of Inflammation, 2018:9471583.

Witchel, S.F., Oberfield, S.E., Peña, (2019). Polycystic Ovary Syndrome: Pathophysiology, Presentation, and Treatment With Emphasis on Adolescent Girls. Journal of the Endocrine Society, 3(8):1545-1573.

Yuan, M., Li, W., Zhu, Y., Yu, B., Wu, J. (2020). Asprosin: A Novel Player in Metabolic Diseases. Frontiers in Endocrinology, 11:64.

Zhang, L., Chen, C., Zhou, N., Fu, Y., Cheng, X. (2019). Circulating asprosin concentrations are increased in type 2 diabetes mellitus and independently associated with fasting glucose and triglyceride. Clinica Chimica act,489:183-188. 